\author{
Alejandro Esguerra ${ }^{1}$ \\ alejandro.esguerra@,uni-bielefeld.de
}

\title{
Zur politischen Epistemologie des Anthropozäns: Epistemische Autorität und institutionelles Design globaler Expertenorganisationen
}

Erscheint in

Sebastian Buettner und Thomas Laux (Hrsg.). „Umstrittene Expertise. Zur Wissensproblematik der Politik“"Leviathan Sonderband 2021

Pre-print Stand 22.7.2021

\section{Einleitung}

Die Geschichte weiß um viele Narrative der sozioökologischen Krise: Das Waldsterben rief in den 1980er Jahren die besondere Beziehung, der Deutschen` zum Wald auf. ${ }^{2}$ Das Ozonloch wurde wenig später sowohl zum Sinnbild globaler Umweltzerstörung mit direkten Effekten für die Unversehrtheit menschlicher Haut als auch zum Beweis der Macht epistemischer Gemeinschaften, gegossen in ein globales Umweltregime. ${ }^{3}$ Im Jahr 2007 erhielt die prominenteste Expertenorganisation, der Weltklimarat (IPCC) den Friedensnobelpreis um ihre Verdienste zur wissenschaftlichen Bestätigung des menschengemachten globalen Klimawandels. ${ }^{4}$

Die Verdichtung der sozioökologischen Krise in der Gegenwart kann mit dem Begriff des Anthropozäns diagnostiziert werden. ${ }^{5}$ Im Gegensatz zu den obigen Beispielen, die einzelnen Probleme in den Vordergrund gerückt haben, bezeichnet das Anthropozän nun die Akkumulation von Problemen, die einen erdsystemischen Effekt auslösen. ${ }^{6}$ Menschliches Handeln selbst ist zum geologischen Faktor geworden. ${ }^{7}$ Es hinterlässt eine Signatur, die möglicherweise stärker als alle anderen physikalischen Kräfte die geologische Geschichte der Erde bestimmen wird. Relativ stabile klimatische Bedingungen und das Vorhandensein natürlicher Ressourcen, Elemente der bisherigen Epoche des Holozäns (seit circa 12.000 Jahren), werden und haben sich bereits im Anthropozän stark verändert. Steigende Temperaturen und Wasserstände gehören zu den sichtbarsten Folgen, die Konflikte um politische Verantwortung entfachen.

\footnotetext{
${ }^{1}$ Ich danke den Herausgebern Sebastian Büttner und Thomas Laux sowie zwei exzellenten anonymen Gutachten für hilfreiche Kommentare und Anregungen. Tobias Berger, Henrike Knappe und Lukas Ziebell haben frühere Versionen dieses Textes hilfreich kommentiert. Zu Dank verpflichtet bin ich auch den Teilnehmenden des Kolloquiums des Institute for Interdisciplinary Studies of Science an der Universität Bielefeld, die wichtige Hinweise zur Rahmung der Argumente gegeben haben.

${ }^{2}$ Das Deutsche Historische Museum in Berlin widmete sich Anfang der 2010er Jahren dem Wald als kulturgeschichtliches Objekt (Quellverweis im Anhang).

${ }^{3}$ Haas 1992.

${ }^{4}$ Beck et al. 2017.

${ }^{5}$ Biermann 2021.

${ }^{6}$ Hamilton 2017.

${ }^{7}$ Crutzen, Stoermer 2000.
} 
Vor diesem Hintergrund fragt der Artikel nach den Konturen einer politischen Epistemologie des Anthropozäns. ${ }^{8}$ Es ist ein Programm, das Wissensbeständen und Epistemologien auf der Spur ist, die das Anthropozän und seine Effekte beschreiben, verdichten oder verneinen; sie werden übersetzt in Lösungen, Zukunftsvisionen und Politiken. Eine solche Epistemologie ist 'politisch' und nicht 'objektiv', da klassische Grenzen, wie die zwischen Wissen und Werten oder Wissenschaft und Politik, nicht als gesetzt, sondern als in Aushandlung konzeptualisiert werden. ${ }^{9}$ Wissensbestände, die beispielsweise den Klimawandel beschreiben, sind kein Produkt abgeschotteter Forschung: Geopolitische Ambitionen veranlasste vor allem die USA zu massiven staatliche Investitionen in geophysische Wissenschaften. Klimawissenschaften entstanden in diesem Zusammenhang eher nebenbei als zielgerichtet. Ihr Entstehungskontext prägt bis heute wie 'Klima' gewusst wird - als globales, geophysisches und nicht als bioökologisches Phänomen. ${ }^{10}$ Eine politische Epistemologie ist politisch, da sie die Mechanismen untersucht, die bestimmtes Wissen relevant für Politik erscheinen lässt und dabei anderes ausgrenzt. Es geht immer um Fragen, welches Wissen, für wen und in wessen Namen produziert und in Governance-Strukturen übersetzt wird. Eine solche politische Epistemologie bricht folglich mit den Annahmen einer ,objektiven' Epistemologie, die nach wie vor in weiten Teilen der Politikwissenschaft beziehungsweise den Internationalen Beziehungen (IB) vorherrschend ist. ${ }^{11}$

Ich nähere mich einer politischen Epistemologie über eine Diskussion zur epistemischen Autorität globaler Expertenorganisationen unter Rückgriff auf Theorien der Science and Technology Studies (STS) und soziologisch inspirierten Arbeiten aus den IB. Dabei fasse ich epistemische Autorität als die autoritative Interpretation politikrelevanten Wissens unter Berücksichtigung exkludierender und selektiver Praktiken; epistemische Autorität stützt sich auf eine Reihe von Legitimationsquellen. ${ }^{12}$ Globale Expertenorganisationen generieren, teils von Staaten beauftragt, politikrelevantes Wissen für drängende Probleme wie Klimawandel oder Biodiversitätsverlust. ${ }^{13}$ Sie sind zentraler Akteur im Wissen um die Probleme des Anthropozäns.

Folgende These möchte ich in diesem Zusammenhang entfalten: Das Anthropozän formt eine drängende, wenn auch diffuse Problemkulisse. In ihrem Streben nach epistemischer Autorität fordert diese Problemkulisse Expertenorganisationen heraus, da die Organisationen sowohl die Probleme in ihrer spezifischen Rahmung selbst mithervorbringen aber gleichzeitig diesen (beispielsweise Klimawandel) auch kaum gerecht werden können. Vor diesem Hintergrund entwickeln Expertenorganisationen neue institutionellen Designs, um relevant für Politik zu werden oder zu bleiben: Lösungsbasierte und zukunftsorientierte Ansätze ${ }^{14}$, Koproduktion ${ }^{15}$ und Pluralisierung von Wissen ${ }^{16}$, verstetigt in institutionellen Designs, konkurrieren nun mit etablierten Normen, wie „policy relevance without being policy prescriptive“ zur Legitimierung epistemischer

\footnotetext{
${ }^{8}$ Zur politischen Epistemologie siehe Straßheim 2015.

${ }^{9}$ Die Science and Technology Studies sprechen hier von „Grenzarbeit“, die in der Koproduktion von Wissen und sozialer Ordnung geleistet wird; siehe dazu Jasanoff 2004.

${ }^{10}$ Allan 2017; Edwards 2010.

${ }^{11}$ Objektive Epistemologien folgen Spielarten des Ansatzes ,,epistemischer Gemeinschaften“, die im Kern konsensuales Wissen und seine Produktion voraussetzen; siehe dazu Haas 1992.

${ }^{12}$ Haas 2018; Beck et al. 2017; Zürn 2018.

${ }^{13}$ Beck et al. 2017.

${ }^{14}$ Haas 2017; Beck, Mahony 2018.

${ }^{15}$ van der Hel 2016.

${ }^{16}$ Esguerra, van der Hel 2021.
} 
Autorität. ${ }^{17}$ Legitimierungspolitiken und die stete Aushandlung was schon als politisch und was noch als wissenschaftlich gilt, sind die Folge. ${ }^{18}$

Um diese Dynamiken zu greifen, entwickelt der Artikel ein prozessuales Verständnis epistemischer Autorität. ${ }^{19}$ Unter Rückgriff auf neuere Arbeiten in den IB sowie den STS soll untersucht werden, wie epistemische Autorität entsteht, sich verfestigt, aber auch wieder verflüssigt. ${ }^{20}$ Expertenorganisationen ringen um Anerkennung und konkurrieren mit anderen Akteuren um Autorität. ${ }^{21}$ Eine dynamische Perspektive erlaubt es zu untersuchen, wie sich dieses Ringen in Verhandlungen in (den) institutionellen Designs niederschlägt. Durch Designs in- und exkludieren Expertenorganisationen Wissensträgerinnen, in- und desintegrieren Wissenssysteme und reorganisieren ihre Kompetenzbereiche im Bestreben, als relevant anerkannt zu werden. ${ }^{22}$ Der Artikel illustriert die Überlegungen anhand dreier, für das Phänomen typischer, Fälle: ${ }^{23}$ „Future Earth" - ein globales Netzwerk zur Nachhaltigkeitsforschung - wird in Hinblick auf die Integration neuer Akteure untersucht. Der „Weltbiodiversitätsrat“ (IPBES) wird als Fall herangezogen, um die Repräsentation unterschiedlichen Wissens zu diskutieren. Der IPCC wird auf die Entwicklung von Anpassungspfaden hin analysiert.

Der Beitrag entwickelt das Argument, indem zunächst das Anthropozän als Problemkulisse beschrieben wird. Anschließend entwickle ich eine dynamische Perspektive auf epistemische Autorität, die es erlaubt zu untersuchen, wie „Future Earth“, IPBES und IPCC institutionelle Designs verhandeln. Nach den empirischen Sondierungen diskutiere ich Implikationen für eine politische Epistemologie des Anthropozäns.

\section{Das Anthropozän als Problemkulisse}

Der Begriff des Anthropozäns ist umkämpft, als analytische wie auch als ontologische Kategorie. Die Erdsystemwissenschaften konzeptualisieren das Anthropozän als einen Bruch, eine qualitative Veränderung in der Funktionsweise des Systems Erde. ${ }^{24}$ Um diesen Bruch deutlich zu machen, schlug der Chemiker Paul Crutzen vor, die offizielle geologische Zeit des Holozäns - das Zeitalter, in dem die Erde über die letzten 10.000 Jahre ein relativ stabiles Klima erfahren hat - für beendet zu erklären. Der Aufbruch in ein neues geologisches Zeitalter ist insofern wichtig zu betonen, als dass es sich nicht um einen Sammelbegriff für die intensivere Nutzung und Zerstörung von lokalen und regionalen Ökosystemen handelt. Vielmehr geht es darum zu verstehen, wie menschliche Aktivitäten selbst zur geologischen Kraft geworden sind, die eine qualitative Veränderung des Systems Erde bewirkt.

\footnotetext{
${ }^{17}$ Esguerra, van der Hel 2021, S. 131; Haas 2018; Beck et al. 2017.

${ }^{18}$ Esguerra et al. 2017.

${ }^{19}$ Dieses dynamische Verständnis von epistemischer Autorität erweitert etablierte Ansätze in den IB zu Expertenwissen von Peter M. Haas 1992. Etablierte Ansätze untersuchen, wie epistemische Gemeinschaften Kooperation zwischen Staaten ermöglichen; epistemische Autorität fungiert hier als das Explanans. Die in diesem Artikel entwickelte Perspektive versteht epistemische Autorität selbst als erklärungsbedürftig. Das heißt, epistemische Autorität wird zum Explanandum.

${ }^{20}$ Krisch 2017.

${ }^{21}$ Sending 2017; Sending 2015.

${ }^{22}$ Beck et al. 2017; Montana 2019; Esguerra, van der Hel 2021.

${ }^{23}$ Seawright, Gerring 2008.

${ }^{24}$ Hamilton 2017, S. 10.
} 
Wann der Beginn des neuen Zeitalters zu datieren ist bleibt umstritten. Das archäologische Argument, das Anthropozän bereits mit der Verwendung von Feuer durch den Menschen beginnen zu lassen, greift zu kurz, da es eben nicht die systemische Signatur des Menschen auf der Erde ausreichend fasst. Die Geographen Simon Lewis und Mark Maslin argumentieren für die Datierung des Anthropozäns auf einen sogenannte Orbis Spike - eine Reduzierung der $\mathrm{CO}_{2}$ Emissionen um 1610. Auslöser war die europäische Invasion Amerikas und den daraus resultierenden Tod tausender indigener Bauern. Ackerland wurde vorübergehend wieder zu Wald, der mehr $\mathrm{CO}_{2}$ absorbieren konnte. Die koloniale Geschichte in die Datierung des Anthropozäns $\mathrm{zu}$ verweben, wird insbesondere von indigen Wissenschaftlerinnen und Wissenschaftlern gefordert. ${ }^{25}$ Crutzen selbst hatte ursprünglich vorgeschlagen, die industrielle Revolution als Marker für das Anthropozän zu setzen. Wesentlicher Bestandteil der industriellen Revolution war der sprunghafte Anstieg des Verbrauchs fossiler Brennstoffe; eine maßgebliche Ursache des menschengemachten Klimawandels. Die industrielle Revolution ging einher mit dem Aufstieg des Kapitalismus, wie Autoren wie Jason Moore hervorheben. ${ }^{26}$ Der Kapitalismus wird in dieser Literatur als zentraler Treiber des Anthropozäns ausgemacht. ${ }^{27}$

Mag die Datierung des Anthropozäns und seine inhaltliche Aufladung umstritten sein, so kann festgehalten werden, dass allen Arbeiten eine gewisse ontologische Annahme gemein ist: Das System Erde ist durch menschliche Aktivitäten bereits jetzt tiefgreifend umgestaltet worden. Verwoben mit wissenschaftlichen Diskursen und soziokulturellen Deutungen, werden die Effekte dieser Umgestaltung konkret; zum einen durch Modelle und Szenarien, zum anderen durch Alltagserfahrungen. (Mikro-) Plastik durchzieht menschliche und nichtmenschliche Körper, teils mit tödlichen, teils mit harmlosen Folgen. Dürreperioden oder der Anstieg des Meeresspiegels als Chiffren des Klimawandels werden übersetzt in global zirkulierende Nachrichten und immer häufiger individuell erfahrbar. In Kalifornien fielen sowohl im Jahr 2020 als auch im Jahr 2021 ganze Siedlungen dem Feuer anheim und färbten den Himmel über San Francisco rot. Ähnliches wird aus Australien berichtet und auch in weiten Teilen Europas und Deutschlands steigt die Zahl der Waldbrände. Jedoch divergieren die Implikationen. Sowohl innerhalb von Gesellschaften als auch zwischen Gesellschaften forciert der Klimawandel soziale Ungleichheit. Verbrannte oder geflutete Häuser können entweder mit Hilfe staatlicher Förderung und eigenem Vermögen wiederaufgebaut werden oder führen zu Armut und Migration.

Die Beispiele verweisen auf das Argument, das ich umreißen möchte: Menschliche Aktivitäten, nun eingeschrieben in das Erdsystem, produzieren Effekte, die sich nur mit hohen Kosten ignorieren lassen. Das Anthropozän und seine Effekte klopfen an und fordern heraus. Sie erzeugen einen „Imperativ“28 , der zwar geleugnet und täglich verdrängt werden kann, dessen Leugnung und Verdrängung jedoch nicht vor den entstehenden Problemen schützt. Anders gesagt, die Beschreibungskategorie Anthropozän zieht eine Reihe von Problemen (Klimawandel, Biodiversitätsverlust, Vermüllung) zusammen, die sowohl in den IB als auch der Soziologie als existentiell beschrieben werden. So sprechen Colgan et al. ${ }^{29}$ in einem prominenten Sonderheft der Zeitschrift ,International Organization“von den „existential politics of climate change“, verstanden

\footnotetext{
25 Tsing et al. 2020.

${ }^{26}$ Moore 2015.

${ }^{27}$ Lövbrand et al. 2020; Burke et al. 2016.

${ }^{28}$ Burke et al. 2016.

${ }^{29}$ Colgan et al. 2021.
} 
als „a contest over whose way of life gets to survive. “30 Klimawandel als existentielles Problem verändert, so die Autoren und die Autorin, Verteilungskämpfe: Staaten, deren Macht und Reichtum auf ihrer Küstenlage fußt, werden strengere Politiken gegen den Klimawandel verabschieden als solche Staaten, die vom Klimawandel politisch und ökonomisch weniger betroffen sind. Diese Dynamik hat Implikationen für das Fortbestehen der globalen liberalen Ordnung.

Soziologisch gewendet und mit Referenzen zu aktuellen Arbeiten in den STS ${ }^{31}$, beschreibt Thomas Scheffer existentielle Probleme als diejenigen Probleme, die die Reproduktion der Gesellschaft in Frage stellen und zur konzertierten Mobilisierung aller Problembearbeitungskapazitäten drängen. ${ }^{32}$ $\mathrm{Zu}$ existentiellen Problemen muss man sich verhalten. Politische Parteien nehmen Stellung in Wahlprogrammen, Unternehmen bemühen sich um Nachhaltigkeitszertifikate, und Museen inkorporieren das Anthropozän in Ausstellungskonzepte. ${ }^{33}$ Nicht zuletzt kreiert die Wissenschaft Wissensinfrastrukturen, um existentielle Probleme wie den Klimawandel beschreibbar zu machen und ihn als spezifisches Problem zu konstruieren. ${ }^{34}$ Neue Institute werden gegründet, die sich nicht nur disziplinär zugeschnitten einer Thematik widmen, sondern Praktiken der Wissensgenerierung in Wissenschaft überhaupt in Frage stellen. Man wird dem Problem (noch) nicht gerecht, es bricht aus und steht doch wieder vor der Tür.

Existentielle Probleme des Anthropozäns bilden eine Problemkulisse, die Expertenorganisationen besonders in Atem hält: So stand der IPCC in der Kritik, da er zwar gemäß seines Mandats den menschengemachten Klimawandel global nachvollzogen hat, jedoch wenig politikrelevantes zu lokalen Anpassungsstrategien vorweisen konnte. ${ }^{35}$ Das Beispiel verweist auf die komplexe Aufgabe von Expertenorganisationen: Ihnen obliegt die autoritative Interpretation relevanten Wissens zu existentiellen Problemen. Dies heißt aber auch, dass diese Organisationen die Probleme in ihrer spezifischen Rahmung mit hervorbringen - ein etabliertes Ergebnis der STS und neuerer Forschung in den IB. ${ }^{36}$ Gleichzeitig können Expertenorganisationen den Problemen auf Grund ihres entdifferenzierenden Charakters immer nur partiell gerecht werden. Stets werden neue Fragen aufgeworfen und die Grenzen zwischen dem, was noch wissenschaftlich oder schon politisch ist, muss immer wieder nachjustiert werden. Dies hat zur Folge, dass die Legitimität epistemischer Autorität auf dem Spiel steht.

\section{Dynamische Perspektiven auf epistemische Autorität}

Um das Ringen um epistemische Autorität zu fassen bedarf es einer Justierung der Analytik. ${ }^{37}$ In den Debatten zur epistemischen Autorität in der Weltpolitik sind die Arbeiten von Haas zu epistemischen Gemeinschaften einschlägig. ${ }^{38}$ Epistemische Gemeinschaften sind Zusammenschlüsse von Expertinnen und Experten die ein Set kausaler und normativer Wirkungszusammenhänge teilen und über eine autoritative Interpretation politikrelevanten Wissens verfügen. Unter Bedingungen von Unsicherheit können epistemische Gemeinschaften Kraft ihrer Autorität die Interessen von Staaten und anderen Akteuren der Weltpolitik

\footnotetext{
${ }^{30}$ Ebd., S. 592.

${ }^{31}$ Beispielsweise Tsing 2015; Latour 2018.

${ }^{32}$ Scheffer, im Erscheinen.

${ }^{33}$ Renn, Scherer 2015.

${ }^{34}$ Edwards 2017; Beck et al. 2017.

${ }^{35}$ Beck et al. 2014.

${ }^{36}$ Allan 2017; Beck et al. 2017.

${ }^{37}$ Die folgenden Überlegungen beziehen sich auf Esguerra, van der Hel 2021.

${ }^{38}$ Haas 1992.
} 
konfigurieren, so dass neue Politiken und Kooperationen entstehen. Mit anderen Worten besteht ein Kausalzusammenhang zwischen epistemischer Autorität (als Explanans) und Kooperation (als Explanandum).

Trotz der enormen Produktivität des Ansatzes, findet sich eine Leerstelle in Bezug auf die Entstehung und Veränderung epistemischer Autorität. Wie gelingt es epistemischen Gemeinschaften sowie anderen Expertinnen- und Experten-Netzwerken sowie -Organisationen als epistemische Autorität anerkannt zu werden? Das heißt: wie können Akteure der Weltpolitik andere davon überzeugen, jenen autoritativen Anspruch auf relevantes Wissen inne zu haben? Diese Fragen sind insofern relevant, da neuere Arbeiten in den IB konturieren, wie das entstandene Global Governance System vor allem auf epistemischer Autorität fußt. ${ }^{39}$ Ein Großteil internationaler Organisationen (IO) hat wenig direkte Sanktionsmöglichkeiten und kann deshalb Staaten und andere Akteure vor allem über Wissensprodukte beeinflussen. Die Überlappung epistemischer Autoritätssphären kreiert zudem eine Situation, in der IOs mit Nichtregierungsorganisationen (NROs) und epistemischen Gemeinschaften um epistemische Autorität konkurrieren und um Anerkennung ringen. ${ }^{40}$ Folglich bedarf es einer Analytik, die die Entstehung und Veränderung beziehungsweise die Verfestigung und Verflüssigung epistemischer Autorität selbst fassen kann. ${ }^{41}$

Zentrales Element einer solchen Analytik ist der Befund, dass sich epistemische Autorität auf unterschiedliche Legitimationsquellen berufen kann. ${ }^{42}$ Etabliert sind Kriterien wie „policy relevance without being policy prescriptive“, „, best available science“ oder „,consensus principle“, die sich vor allem in den letzten 30 Jahren im Kontext des IPCCs entwickelt haben. ${ }^{43}$ Diese Legitimationsquellen zielen darauf ab, wissenschaftliches Wissen als technisch und politikfern zu rahmen bei gleichzeitiger Relevanz für Politik. Dem entgegengesetzt finden sich auch auf internationaler Ebene Legitimationsquellen, die verstärkt die Inklusion unterschiedlicher Wissenssysteme beschreiben. Neure Arbeiten stellen deshalb heraus, dass vermehrt Spannungen zwischen Legitimationsquellen, also Legitimationspolitiken auftreten. ${ }^{44}$

In den STS hat sich etabliert, epistemische Autorität selbst in den Blick zu nehmen (epistemic authority in the making). Die entwickelten Prozessperspektiven zielen stärker als politikwissenschaftliche Arbeiten auf die Selektionsmechanismen und Grenzziehungen in der Produktion politikrelevanten Wissens $a b .{ }^{45}$ Zentrale Fragen lauten dann: „, who belongs within the relevant expert collective and, hence, is entitled to speak for it, as well as who does not belong and, hence, lacks such authority." ${ }^{36}$ Veränderungen in der Wissensproduktion verschärfen die ohnehin umkämpften Arenen der Generierung relevanten Wissens. Zentraler Fokus der Analyse bilden die institutionellen Dynamiken und Designs, in denen Wissen autorisiert wird. ${ }^{47}$ Im Gegensatz zu Arbeiten der politikwissenschaftlichen Assessment-Literatur, die Legitimitätsanforderungen an

\footnotetext{
${ }^{39}$ Zürn 2018

${ }^{40}$ Sending 2017; Faude, Fuß 2020.

${ }^{41}$ Krisch 2017.

${ }^{42}$ Haas 2018.

${ }^{43}$ Diese Kriterien werden diskutiert u.a. von Keller 2010; Beck et al. 2017, S. 1070; Haas 2018.

${ }^{44}$ Haas 2018; Esguerra, van der Hel 2021.

45 Jasanoff 2004.

${ }^{46}$ Beck et al. 2017, S. 1086.

${ }^{47}$ Miller 2017.
} 
bestimmte fixe Designelemente koppelt, ${ }^{48}$ untersucht eine dynamische Perspektive, wie institutionelle Designs verhandelt werden und sich ändern, damit Organisationen als Autorität anerkannt werden. Institutionelle Designs definieren Machtbeziehungen zwischen Teilnehmenden. ${ }^{49}$ Sie sind zudem eingebettet in tiefsitzende Machtstrukturen globaler Wissenschaft sowie - im Falle von intergouvernementalen Organisationen - in die Regeln diplomatischer Praxis. ${ }^{50}$

\section{Ringen um epistemische Autorität: Institutionelle Designs globaler Expertenorganisationen}

Globale Expertenorganisationen sehen sich komplexen Herausforderungen ausgesetzt: Organisationen wie der IPCC haben in den letzten 30 Jahren institutionelle Designs entwickelt, die es ihnen ermöglicht haben, sowohl relevant für Politik zu sein, bei gleichzeitiger Aufrechterhaltung ihrer wissenschaftlichen Glaubwürdigkeit. ${ }^{51}$ Mit dieser Strategie gelang es beispielsweise dem IPCC, existentielle Probleme des Anthropozäns zu erforschen. Doch der drängende Charakter existentieller Probleme erlaubt keinen Stillstand. Für Expertenorganisationen bedeutet dieser Sachverhalt ein Ringen um Anerkennung, der sich in den institutionellen Designs widerspiegelt.

Drei Entwicklungen sind zu beobachten: die Pluralisierung von Wissensakteuren, die Integration unterschiedlicher Wissenssysteme sowie die Schaffung von antizipativem Wissen. Gemein ist diesen drei Entwicklungen, dass sie im Konflikt mit etablierten Normen zur Legitimierung epistemischer Autorität stehen. Im Folgenden zeichne ich den Umgang mit den entstehenden Konflikten in drei unterschiedlichen Expertenorganisationen nach.

\subsection{Pluralisierung der Wissensakteure: Future Earths Institutionalisierung von Ko-produktion}

Die Pluralisierung von Wissensakteuren wird häufig unter dem Begriff der Koproduktion verhandelt. In seiner Praxis-Interpretation versteht man unter Koproduktion, dass neben Wissenschaftlerinnen auch Betroffene beziehungsweise Praxispartner in die Produktion von Wissen eingebunden werden. ${ }^{52}$ Ähnlich beschreibt Karin Bäckstrand mit dem Schlagwort der „Civic Science“ unterschiedliche Praktiken, denen gemein ist, die öffentliche Teilnahme in der Produktion wissenschaftlichen Wissens zu erhöhen. ${ }^{53}$ Die Rechtfertigung für Praktiken der Koproduktion oder „Civic Science“ speist sich aus einer Reihe unterschiedlicher Normen. ${ }^{54}$ Während sich zu Beginn dieses Jahrtausends Konflikte beispielsweise im IPCC in erster Linie an geographischer Teilhabe entzündeten, lässt sich zunehmend beobachten, dass von diversen Stakeholdern Repräsentation eingefordert wird.

Die Forschungsplattform „Future Earth“ gehört zu den ersten Expertenorganisationen, die Koproduktion prominent auf internationaler Ebene institutionell verankert haben. „Future Earth“

\footnotetext{
${ }^{48}$ Mitchell et al. 2006.

${ }^{49}$ Montana 2019.

${ }^{50}$ Folkers 2020; Hughes, Vadrot 2019.

${ }^{51}$ Keller 2010.

52 Beck 2019.

${ }^{53}$ Bäckstrand 2003.

${ }^{54}$ van der Hel 2016.
} 
wurde 2012 mit dem Ziel initiiert, die Interaktion zwischen Wissenschaft und Politik für nachhaltige Entwicklung zu stärken und einen „new social contract“ zwischen Wissenschaft und Gesellschaft zu etablieren. ${ }^{55}$ Aufbauend auf eine Reihe von Vorgängerorganisationen (den sogenannten „Global Change“-Programmen) der vergangenen Jahre Stand zur Debatte, wie sich eine neue Forschungsplattform positionieren könnte, um sowohl innerhalb der Wissenschaftsgemeinschaft als auch seitens Politik und Gesellschaft als legitim anerkannt zu werden. Zentral innerhalb dieses Positionierungsprozesses der entstehenden Organisation war die Betonung eines lösungsorientierten Ansatzes (solutions-orientated science) mit Praktiken von Koproduktion als innovatives und strategisches Element. ${ }^{56}$

Koproduktion steht hier für die Einbeziehung gesellschaftlicher Akteure in der Generierung politikrelevanten Wissens. ${ }^{57}$ Während sich Praktiken der Koproduktion bereits in den letzten Jahrzenten entwickelt haben, kann „Future Earth“ für sich reklamieren, Koproduktion prominent auf globaler Ebene institutionalisiert zu haben. Mit anderen Worten geht es „Future Earth“ nicht nur darum, lokale, vereinzelte Projekte zu fördern, sondern vielmehr Koproduktion als Prinzip der global agierenden Organisation selbst hervorzuheben. Mit Sheila Jasanoff kann davon ausgegangen werden, dass solche Veränderungen in der Generierung wissenschaftlichen Wissens die Fundamente wissenschaftlicher Autorität berührt und folglich Konflikte produziert. ${ }^{58}$ Diese Konflikte werden beobachtbar in den Verhandlungen zur Institutionalisierung von Koproduktion.

In den Verhandlungen zur Ausgestaltung des institutionellen Designs von „Future Earth“ wurde neben einem „Science Committee“ (ein etabliertes Organ in internationalen Forschungsprogrammen) auch ein „Engagement Committee“ eingesetzt. ${ }^{59}$ Während ein „Science Committee“ mit anerkannten Wissenschaftlerinnen und Wissenschaftlern besetzt ist, um die Forschungsrichtlinien zu bestimmen, sollte das „Engagement Committee“ dazu dienen, nichtwissenschaftliche Akteure einzubinden. Kontrovers diskutiert wurde, welches der beiden Komitees in der institutionellen Hierarchie mit mehr Macht versehen werden sollte. So argumentierten einige Gründungsmitglieder, dass das neue Forschungsprogram direkt den Wissensanforderungen von Gesellschaften entsprechen sollte und deshalb das „Engagement Committee“ hierarchisch höher im institutionellen Design einzustufen sei. Dieser Sicht wurde mit Verweis auf die Norm der wissenschaftlichen Autonomie heftig widersprochen. Eine duale Struktur auf Augenhöhe sollte den Konflikt lösen und sicherstellen, dass Repräsentantinnen und Repräsentanten aus der Gesellschaft eine klare institutionelle Verankerung haben. ${ }^{60}$ Ein Verhandlungsteilnehmer beschreibt die Lösung mit den Worten: „... to have a place where you have your policy-relevance and you have your stakeholders, and then at the same time scientists also have some space to meet separately. ${ }^{61 "}$

Jedoch riss die Kritik an der dualen Struktur nicht ab. Mit der Etablierung einer Zwei-Kammer Lösung sei de facto die ,Wissenschaft" abgeschirmt und das „Engagement Committee“ mit

\footnotetext{
${ }^{55}$ Kershaw 2018, S. 123.

${ }^{56}$ van der Hel 2016; Leemans 2016.

${ }^{57}$ Miller, Wyborn 2018; Beck 2019.

58 Jasanoff 2006.

${ }^{59}$ van der Hel 2016, S. 170.

${ }^{60}$ Leemans 2016.

${ }^{61}$ Zitiert in van der Hel 2016, S. 171.
} 
partizipativen Projekten befasst. ${ }^{62}$ Nach einigen Jahren der operativen Differenzierung wurde 2018 die entwickelte Struktur zu Gunsten eines einzigen „Advisory Committee“ aufgehoben. Die Besetzung des Gremiums deutet allerdings an, dass dessen Mitglieder stark in der Wissenschaft verankert sind. ${ }^{63}$ Ähnlich wie die Untergliederungen der Organisation, verändert sich auch das Leitungsgremium - "The Governing Council" - in Ausrichtung und Mitgliedschaft. Sollten zunächst die initiierenden Akteure einem Multi-Stakeholder Gremium, bestehend aus "scientists, policymakers, development actors, representatives of business and industry, civil society and other stakeholders" weichen, so wurde 2018 entschieden, vor allem etablierte Organisationen in das Council zu wählen. ${ }^{64}$ Neben internationalen Wissenschaftsorganisationen indiziert die Beteiligung des United Nations Environmental Programme (UNEP) die Rückbindung Future Earths an politische Prozesse. $^{65}$

Ein weiteres institutionelles Instrument um unterschiedliche Wissensakteure an der Plattform zu beteiligen und Koproduktion zu ermöglichen sind die entwickelten „Knowledge Action Networks“. Die Netzwerke vereinen, so die Organisation, "Innovatoren" aus allen Teilen der Gesellschaft. ${ }^{66}$ Die Funktion, der zur Zeit acht thematischen Projekte, bleibt jedoch die Generierung von „high-quality actionable scientific knowledge“ bei gleichzeitiger Beteiligung gesellschaftlicher Partnerinnen und Partner, um relevantes Wissen zu erstellen. ${ }^{67}$ Auch aufgrund fehlender formaler Regeln bestehen die Netzwerke im Gegensatz zu den präsentierten Ambitionen in erster Linie aus Wissenschaftlerinnen und nicht Praktikern.

Zusammenfassend läßt sich festhalten, dass in der Entstehungsgeschichte von „Future Earth“ die Institutionalisierung von Koproduktion eine zentrale Rolle gespielt hat, um den Herausforderungen des Anthropozäns zu begegnen und damit einhergehend die Organisation als relevant zu kennzeichnen. ${ }^{68}$ Die diskutierten Designoptionen zeigen allerdings an, wie unterschiedliche Vorstellungen davon existieren, was Koproduktion genau beinhaltet und wie es $\mathrm{zu}$ institutionalisieren ist. ${ }^{69}$

\subsection{Repräsentation unterschiedlichen Wissens: IPBESs kon₹eptuelle Rahmung}

Expertenorganisationen repräsentieren verstärkt unterschiedliche Wissenssysteme. Organisationen schaffen entweder implizit oder explizit konzeptuelle Rahmungen, die Wissensprodukten wie Assessments eine Form geben und die Arbeit der Organisation über Jahre hinweg bestimmen können. Folglich sind konzeptuelle Rahmungen umkämpft, auch weil sie im Erringen epistemischer Autorität essentiell sind. Konflikte um Repräsentation lassen sich am „Conceptual

\footnotetext{
${ }^{62}$ Esguerra, van der Hel 2021, S. 138.

${ }^{63}$ Future Earth 2018.

${ }^{64}$ Future Earth 2013.

${ }^{65}$ Die Organisationen sind: "International Science Council” (ISC), "The Belmont Forum of Funding Agencies", "The United Nations Educational, Scientific, and Cultural Organization" (UNESCO), "The United Nations Environment Programme" (UNEP), "The United Nations University” (UNU), "Sustainable Development Solutions Network" (SDSN), "The STS Forum" und die "World Meteorological Organization".

${ }^{66}$ Future Earth 2021.

67 ebd.

${ }^{68}$ Beck 2019.

${ }^{69}$ van der Hel 2016.
} 
Framework“"von IPBES nachzeichnen, welches ein zentrales Element im Positionierungsprozess der Organisation war.

Gegründet 2012, entwarf sich IPBES mit dem Slogan „An IPCC for Biodiversity ${ }^{\text {“70 }}$ als direkte Kopie der erfolgreichen Schwesterorganisation und versuchte gleichzeitig Anerkennung zu erlangen, indem innovative und reflexive Elemente betont wurden. ${ }^{71}$ Zentraler Bestandteil der Innovation war das entworfene „Conceptual Framework“, dessen Aushandlung zwei diametral entgegengesetzte Verständnisse von Biodiversität verdeutlicht. In den letzten Jahren wurde Biodiversität insbesondere von Ökonominnen und Ökologen vermehrt als Ökosystemdienstleistung (eco-system services) gerahmt und auch als solche in der Politik kommuniziert. Das Konzept erlaubt es, Ökosysteme ökonomisch zu bewerten und den Verbrauch dieser Werte zu kritisieren. ${ }^{72}$ Auch der Wert eines Waldspaziergangs lässt sich so monetarisieren.

Während das „Konzept der Ökosystemdienstleistung“ bereits die Organisation selbst beschreibt „The Intergovernmental Platform on Biodiversity and Ecosystem Services - stieß die Rahmung in den Verhandlungen zum „Conceptual Framework“ auf Widerstand. Sozialwissenschaftliche Forschung problematisierte ein Verständnis von Biodiversität, das ,Natur von vorneherein als Markt und Dienstleistung engführe; alternative Verständnisse würden durch diese Rahmung ausgeschlossen. ${ }^{73}$ Insbesondere die bolivianische Delegation, die unter anderem durch einen promovierten Anthropologen geleitet wurde, sprach sich in den Verhandlungen vehement für ein holistisches Konzept aus, das indigene Verständnisse von Natur anerkennen würde. Ähnlich der bolivianischen Verfassung sollten Begriffe wie „living-well in balance and harmony with Mother Earth“ das „Conceptual Framework“ leiten. ${ }^{74}$

In den Verhandlungen beschuldigten sich Verfechterinnen und Verfechter beider Positionen keine wissenschaftliche, sondern eine politische Diskussion zu führen. ${ }^{75}$ Der Konflikt durchzog mehrere Workshops, in denen die Teilnehmenden zwar intensiv diskutiert und das Framework ständig veränderten, jedoch nicht bereit waren, ihre Positionen aufzuweichen. Der geschaffene Kompromiss präsentiert nun beide Sichtweisen, Seite an Seite, visualisiert durch eine Grafik. In der Unterschrift heißt es:

In each of the boxes, the headlines in black are inclusive categories that should be intelligible and relevant to all stakeholders involved in IPBES and embrace the categories of western science (in green) and equivalent or similar categories according to other knowledge systems (in blue). ${ }^{76}$

Maud Borie und Michael Hulme sprechen von einem „consensus out of dissensus ${ }^{\text {“77 }}$, der durch eine blaue Kodierung für den „Mother Earth“-Ansatz und eine grüne Kodierung für den Ansatz der Ökosystemdienstleistung zwar Einigung ermöglicht, jedoch möglicherweise keine Integration schafft. Diese Konflikte um Repräsentation verschärfen sich unter anderem auch deshalb, weil der Dominanz naturwissenschaftlichen Wissens mit sozial- und humanwissenschaftlichem neben indigen und lokalem Wissen entgegengewirkt werden soll. Detaillierte Regeln für die Besetzung

\footnotetext{
${ }^{70}$ Nature 2010.

${ }^{71}$ Beck et al. 2014.

72 Nadim 2016.

73 Turnhout et al. 2013.

${ }^{74}$ Plurinational State of Bolivia 2014.

${ }^{75}$ Borie, Hulme 2015.

${ }^{76}$ Díaz et al. 2015.

${ }^{77}$ Borie, Hulme 2015, S. 493.
} 
von Expertengremien zeigen an, dass höchst unterschiedliche Wissenssysteme integriert und durch die Organisation repräsentiert werden sollen. Diese Repräsentation der Heterogenität wird als eine Anstrengung interpretiert, epistemische Autorität zu erzielen. ${ }^{78}$

\subsection{Zukunftsobjekte: IPCCs Anpassungspfade}

Die beschriebenen Veränderungen in der Generierung politikrelevanten Wissens haben bereits Innovationen im 'wer' und 'wie' indiziert. Doch auch, was' als relevantes Wissen angesehen wird ist im Wandel begriffen. Der „Wissenschaftliche Beirat der Bundesregierung Globale Umweltveränderungen“ (WBGU) hat bereits in seinem 2011er-Gutachten „Große Transformation“ für neue Formen des Wissens geworben, nicht zuletzt für antizipatives Wissen, also solches Wissen, das mögliche Zukünfte beschreibt. ${ }^{79}$ Antizipatives Wissen wird in einer Reihe von Expertenorganisationen unter anderem auch bei „Future Earth“ und IPBES diskutiert. ${ }^{80}$ Das prägnanteste Beispiel findet sich jedoch in den Veränderungen innerhalb des IPCCs, der sich zur Sicherung seiner Autorität neuen Aufgaben zugewandt hat.

Um die neuen Formen des Wissens zu verstehen, sei zunächst auf die historische Rolle des IPCCs verwiesen, der sich bei seiner Gründung 1988 in die multilaterale Ordnung mit Staaten als Adressatinnen von konsensualem Expertenwissen einfügte. ${ }^{81} \mathrm{Um}$ als epistemische Autorität bezüglich des Klimawandels anerkannt zu werden, entwickelte der IPCC eine Reihe von Normen wie das Konsensprinzip und entschied, Klimawandel insbesondere als globales Phänomen zu rahmen. ${ }^{82}$ Diese Strategie ermöglichte es, den anthropogenen Klimawandel als wissenschaftlichen Konsens zu etablieren. Eine Errungenschaft, die nicht zuletzt 2007 mit dem Friedensnobelpreis belohnt wurde.

Mit dem „Pariser Abkommen“ von 2015 und den damit einhergehenden Fragen nach nationaler Implementierung und internationaler Überwachung von Klimaanpassungsmaßnahmen hat der IPCC begonnen, einen lösungs- und zukunftsorientierten Ansatz zu entwickeln, in dem „Integrierte Assessment Modelle“ (IAM) eine zentrale Rolle spielen. ${ }^{83}$ Ursprünglich wurden mit den IAM Szenarien entwickelt, die anzeigen konnten, wie Emissionen durch plausible ökonomische und technologische Entwicklungen beeinflusst werden würden. ${ }^{84}$ Die Szenarien zeichneten unterschiedliche Emissionspfade nach, die es erlaubten, Policy-Makern unterschiedliche Welten zu präsentieren, in denen bestimmte Klimapolitiken greifen um so Kosten und Nutzen ihrer politischen Entscheidungen abzuwägen. ${ }^{85}$

Die neuen IAM haben diese Logik umgedreht. Es werden nun nicht mehr Emissionen anhand von plausiblen Annahmen modelliert, sondern es werden mögliche Pfade konstruiert um zu verstehen, wie bestimmte Klimaziele erreicht werden können; die Differenz wird semantisch dadurch gekennzeichnet, dass von Anpassungspfaden und nicht mehr von Emissionspfaden gesprochen

\footnotetext{
${ }^{78}$ Gustafsson, Lidskog 2018.

${ }^{79}$ WBGU 2011, S. 341; Knappe et al. 2019

${ }^{80}$ Beck 2019; Beck, Forsyth 2020.

${ }^{81}$ Beck et al. 2017.

${ }^{82}$ Edwards 2010.

${ }^{83}$ Beck, Mahony 2018.

${ }^{84}$ Beck, Oomen, 2021.

${ }^{85}$ Pielke 2018.
} 
wird. ${ }^{86}$ Diese neue Zukunftsorientierung hat eine Reihe von Implikationen, von denen zwei hervorzuheben sind: Zum einen kann der Bruch mit der bisherigeren Praxis daran abgelesen werden, dass Kontroversen entstanden, ob der IPCC möglicherweise mit seiner Rolle als Begutachter bestehenden Wissens und somit selbst zum Produzenten von Wissen werden würde. ${ }^{87}$ Um seiner etablierten Rolle gerecht zu bleiben, bedurfte es vorsichtiger Reorganisation: Anstelle selbst Anpassungspfade zu entwerfen, wurde die IAM Community beauftragt, Szenarios entlang bestimmter Zielvorgaben zu entwickeln; diese Ergebnisse konnten dann wiederum begutachtet werden, so dass der IPCC seiner Funktion als Assessmentorganisation treu blieb.

Zum anderen sind die entwickelten „Representative Concentration Pathways“ (RCP) politisch performativ, da sie eine Vision von Zukunft entwerfen, die politische Einigung in der Gegenwart ermöglicht. ${ }^{88}$ Silke Beck und Martin Mahony arbeiteten heraus, dass das Pariser Abkommen von 2015 mit dem gesteckten Ziel unter die kritische Zwei-Grad-Marke zu kommen durch politisch koproduzierte RCPs zustande gekommen ist. ${ }^{89}$ Insbesondere der RCP2.6 entwarf einen umstrittenen Anpassungspfad, der das ambitionierte Ziel im Reich des Möglichen verortete, ohne einen zu radikalen Wandel zu postulieren. Der Pfad setzt auf die Effekte von sogenannten negativen Emissionstechnologien (NET). NET gehören zu einem Cluster von Technologien, die darauf setzen, die klimatischen Veränderungen durch technologische Innovation abzufedern. Dabei reichen die vorgeschlagenen Projekte von der Einlagerung von $\mathrm{CO}_{2}$ unter der Erde bis über die Reduzierung der Sonneneinstrahlung durch Reflektoren im Weltraum.

Dieses Cluster von Technologien ist umstritten, da ihre negativen Effekte kaum erforscht sind und sie gleichzeitig über sogenannte „Techno-Fixes“ suggerieren, die dramatischen Folgen des anthropogenen Klimawandels in den Griff bekommen zu können. ${ }^{90}$ Das Problem wirkt umso schwerer, da unklar ist, inwieweit sich an ambitionierte Anpassungspfade interessierte PolicyMaker aus der EU den Implikationen der Technologien bewusst waren und sind. ${ }^{91} \mathrm{Da}$ die Diskussion um die Technologien insbesondere von Wissenschaftlerinnen und Wissenschaftlern des globalen Nordens geführt wird, wird der globale Süden im Entwerfen von Zukünften marginalisiert. ${ }^{92}$ So muss der globale Süden nicht nur historisch die negativen Effekte des Anthropozäns mit tragen, sondern bekommt gleichzeitig im Diskurs kein Mitspracherecht darüber zugebilligt, wie die Folgen des Klimawandels zu bewältigen sind. ${ }^{93}$ Das Entwerfen von Zukünften ist kein apolitisches Imaginieren; es schafft vielmehr Handlungskorridore und Pfadabhängigkeiten, die global Politik strukturieren.

\subsection{Epistemische Autorität globaler Expertenorganisationen im Anthropozän}

Betrachtet man nun alle drei Fälle gemeinsam, so lässt sich feststellen, dass epistemische Autorität im Anthropozän weniger als gegeben oder delegiert, sondern als stets neu ausgehandelt, das heißt

\footnotetext{
${ }^{86}$ Anderson, Jewell 2019.

${ }^{87}$ Beck, Mahony 2018.

${ }^{88}$ Esguerra 2019

${ }^{89}$ Beck, Mahony 2018

${ }^{90}$ Haraway 2016

${ }^{91}$ Beck, Mahony 2018

${ }^{92}$ Biermann, Möller 2019

${ }^{93}$ Yusoff 2018
} 
dynamisch zu theoretisieren ist. Diese Dynamik liegt in der Konkurrenz von Legitimitätsnormen begründet. Etablierte Normen der Wissenschafts- und Weltpolitik entwerfen ein Narrativ, nach dem wissenschaftliches, konsensuales Wissen Staaten befähigen soll, angemessene Politik zu verfolgen. ${ }^{94}$ Diese klassischen Konzeptionen sind eingebettet in tiefsitzende Machtstrukturen globaler Wissenschaft sowie internationaler Politik. ${ }^{95}$ So sind es nicht zuletzt Staaten und nicht NROs, die Stimmrecht in IOs besitzen; von disziplinären Expertinnen und Experten begutachtetes, in internationalen Zeitschriften veröffentlichtes Wissen, gilt als robust im Gegensatz $\mathrm{zu}$,grauer Literatur'.

Die drei eingeführten Fälle reflektieren diese etablierten Normen und weisen gleichzeitig über sie hinaus. Die diskutierten Expertenorganisationen befähigen neben Staaten auch andere Akteure sich in Nachhaltigkeitspolitik einzubringen und ihrer Stimme auf internationaler Bühne Gehör zu verschaffen; nicht ausschließlich konsensuales Wissen sondern multiple Problemrahmungen und Lösungen erscheinen en vogue. ${ }^{96}$ Die Kopräsenz widerstreitender Legitimitätsgrundlagen, institutionalisiert im Design der Organisationen, erfordert Aushandlung.

Das beschriebene Spannungsverhältnis zwischen etablierten und neu entstehenden Normen zur Legitimierung epistemischer Autorität birgt aber auch Potential. Eleanor Kershaw zitiert eine Interviewpartnerin in "Future Earth” mit den Worten, „I mean, one really good thing is even having discussions about [power relations], because in somewhere like the IGBP Science Committee I can assure you the notion of talking about power would never have come up [laughs]. ${ }^{\text {(997 }}$ Machtasymmetrien, konkurrierende Repräsentationen und Zukunftsvisionen werden durch die beschriebenen Veränderungen in Expertenorganisationen selbst, sowie in der rezipierenden Öffentlichkeit thematisch. Dieses reflexive Moment scheint insofern angebracht, da den existentiellen Problemen des Anthropozäns eben noch keine angemessenen Institutionen zur Seite gestellt sind.

\section{Implikationen für eine politischen Epistemologie des Anthropozäns}

Die Diskussion zu epistemischer Autorität insbesondere von Expertenorganisationen kann in ein größeres Programm eingebettet werden, das vorläufig als politische Epistemologie des Anthropozäns umrissen werden kann. Eine politische Epistemologie grenzt sich zunächst von einer objektiven Epistemologie - immer noch vorherrschend in der Politikwissenschaft - durch folgende Aspekte ab: ${ }^{98}$ Erstens wird die klare Unterscheidung zwischen Wissen und Werten zugunsten einer Rekonstruktion der Interaktion und Implikationen aufgehoben. Die Diskussion über die konzeptuelle Rahmung des IPBES hat gezeigt, wie konkurrierende Rahmungen von Biodiversität, die jeweils andere Rahmung als wertbehaftet und diskussionswürdig beschreibt. Objektivitätsforderungen können aus der Perspektive einer politischen Epistemologie als Strategie untersucht werden, eigene Deutungsmuster durchzusetzen. ${ }^{99}$

\footnotetext{
${ }^{94}$ Haas 1992; Esguerra 2015

${ }^{95}$ Folkers 2020; Hughes, Vadrot 2019

96 Turnhout et al. 2012; Maas et al. 2021

${ }^{97}$ zitiert in: Kershaw 2018, S. 125

${ }^{98}$ siehe dazu Straßheim 2015, S. 322

${ }^{99}$ Haraway 1988
} 
Zweitens versteht eine politische Epistemologie die Grenze zwischen Wissenschaft und Politik nicht als gesetzt, sondern geht vielmehr davon aus, dass Akteure sich in ständiger „Grenzarbeit“ befinden. ${ }^{100}$ Der Grenzziehung selbst gilt das analytische Interesse. Die vom IPCC begutachteten Anpassungspfade sind Beispiele für ein nuanciertes Austarieren, um als politikrelevant und gleichzeitig nicht als Politik vorschreibend wahrgenommen zu werden. Die Konstruktionsarbeit an Grenzziehungen zu untersuchen hat nicht zum Ziel, die Grenzziehung an sich zu delegitimieren. Das Erkenntnisinteresse einer politischen Epistemologie ist vielmehr, die Bedingungen zu erforschen, unter den Gesellschaften Wissen als autoritativ anerkennen.

Drittens bricht eine politische Epistemologie mit der normativen Tendenz vieler objektiver Epistemologien, die Rationalisierung von Politik durch Wissenschaft generell als erstrebenswert zu theoretisieren. Angesicht existenzieller Probleme wie dem Klimawandel oder Viruspandemien, scheint der Ruf nach Expertokratien zunächst angemessen, um Politiken nach wissenschaftlichen Erkenntnissen auszurichten. Eine politische Epistemologie verweist jedoch auf die Komplexität geo-sozialer Veränderungen. Um überhaupt Modelle $\mathrm{zu}$ entwickeln, die beispielsweise Klimaveränderungen darstellen, haben Wissenschaftlerinnen und Wissenschaftler in den letzten Jahrzehnten gelernt, mit Unsicherheiten produktiv umzugehen. ${ }^{101}$ Es wird interpretiert, ausgeblendet, geschätzt und übersetzt. Diese wissenschaftliche Praxis hat Implikationen. Sie führt im besten Fall zu einer Form der „,humble science“ ${ }^{\text {102 }}$, die sich ihrer eigenen Situierung bewusst ist und diese entsprechend kommuniziert. Der „Future Earth“-Ansatz der Koproduktion kann als Versuch interpretiert werden, Räume der Reflexion zu schaffen, wie eine Beteiligte beschreibt: Eleanor Kershaw zitiert eine Interviewpartnerin von "Future Earth" mit den Worten:

I would see the sort of co-construction agenda as about people (...) coming to a situation bringing their own social commitments and drivers and understandings and assumptions about the world, and bringing those together and reflecting on them reflexively, realising that everybody's got a set of partial perspectives so those need to be deliberated on. ${ }^{103 ،}$

Eine politische Epistemologie untersucht, wie in diesen Situationen Politik und Wissenschaft verhandelt werden. ${ }^{104}$ Politische Kämpfe um autoritative Setzungen stehen im Mittelpunkt der Analyse.

Eine politische Epistemologie des Anthropozäns untersucht Wissensbestände, die die Problemkulisse existentieller Probleme hervorbringen aber eben auch negieren. Der drängende und anklopfende Charakter existentieller Probleme hält, wie gezeigt, Expertenorganisationen in ihren Anstrengungen in Atem, gesellschaftliche und politische Relevanz zu erlangen. Die Konkurrenz umfasst ein weites Spektrum: Leugnerinnen und Leugner existentieller Probleme verneinen Klimawandel oder Pandemie und erschaffen teils erstaunlich wirkmächtige Ideologien. Advokatinnen und Advokaten von „technological fixes“ ${ }^{\text {(105 }}$ entwerfen, wie aktuelle Diskussionen zu NET zeigen, Visionen, die die Anthropozän These ad absurdum führen: Im Sinne einer Herrschaft durch Technik wird, der durch die Anthropozän These dokumentierte, nicht-

\footnotetext{
100 Beck et al. 2017

${ }^{101}$ Gramelsberger, Feichter 2011

102 van der Hel 2016

${ }^{103}$ Kershaw 2018, S. 121

${ }^{104}$ Eine politische Epistemologie ist deshalb verwandt mit einem analytischen Verständnis von Ko-produktion Jasanoff 2004. Dies geht davon aus, dass Wissenschaft und Gesellschaft schon immer ko-produziert sind und die Frage nur ist, wie das Verhältnis konkret ausgestaltet ist.

${ }^{105}$ Haraway 2016
} 
intendierte menschliche Einfluss auf geologische Prozesse zum Anlass genommen, nun intendierte Effekte durch technologische Experimente zu erzielen. Doch beruht das Gros der negativen Emissionstechnologien weder auf wissenschaftlichen Evidenzen noch wird die problematisierende sozialwissenschaftliche Literatur rezipiert. ${ }^{106}$ Konkurrenz erhalten Expertenorganisationen zudem von sozialen Bewegungen und ihren Verbündeten. Sie verweisen auf die Verschränkungen klassischer Wissenschaft - auch der Geologie - mit (neo-)kolonialen und (neo-)liberalen Strukturen und problematisieren existierende Rahmungen, auch die des Anthropozäns selbst, die nicht ausreichend historisch produzierte Ungleichheiten reflektieren. ${ }^{107}$ Soziale Bewegungen und NGOs nutzen die sich öffnende Expertenorganisationen als Bühne, um ihre Anliegen zu artikulieren.

Folglich muss sich eine politische Epistemologie des Anthropozäns neben den diskutierten Expertenorganisationen auch weiteren Praktiken, Institutionen und Diskursen widmen, die versuchen das Anklopfen existentieller Probleme zu beantworten. Eine zentrale Herausforderung ist dabei, die ontologische Gegebenheit existentieller Probleme zu theoretisieren. Reflexartig wird untersucht, wie Probleme zu Problemen gemacht werden, wie sie unterschiedliche gewusst und beantwortet werden können. Eine politische Epistemologie des Anthropozäns interessiert sich für diese Wissensbestände und versucht gleichzeitig die ,neue Verwobenheit des Menschen' und die resultierenden Effekte ernst zu nehmen. Relevante sozial- und geisteswissenschaftliche Diskurse zum Anthropozän verweben in den relationalen Teppich menschlicher Beziehungen, nichtmenschliche Akteure (non-humans). ${ }^{108}$ Dabei geht es weniger darum Artefakten Akteursqualität einzuhauchen, sondern vielmehr um die massive Abhängigkeit des Menschen vom System Erde. ${ }^{109}$

\section{Konklusion}

Der Begriff des Anthropozäns ist sowohl produktiv als auch höchst umstritten. Denkt man das Anthropozän als eine Sammlung existentieller Probleme, wie Klimawandel oder Biodiversitätsverlust, die den Fortbestand von Gesellschaften in Frage stellen, wird der drängende Charakter des Anthropozäns deutlich. Der Klimawandel als gegebenes Problem, das unterschiedlich verstanden, verdrängt oder geleugnet werden kann, sich dennoch immer wieder repräsentiert, fordert Expertenorganisationen heraus; nicht zuletzt, da sie die spezifische Rahmung der Probleme selbst entwerfen. Expertenorganisationen bringen neue Designs hervor und reorganisieren ihre Ausrichtung. Ich habe dies an drei Beispielen illustriert: Die Pluralisierung von Wissensakteuren, die Repräsentation unterschiedlichen Wissens sowie die Erschaffung von Zukunftsobjekten zeigen an, dass Institutionen, die nach epistemischer Autorität streben, in Bewegung sind. Die Beispiele betreffen nicht nur die angeführten Organisationen und Netzwerke sondern lassen sich sowohl in den jeweils anderen hier diskutierten Organisationen beobachten ${ }^{110}$ als auch in weiteren Expertenorganisationen. ${ }^{111}$ Die Suche nach neuen Rollen beziehungsweise Legitimationen ist keineswegs konsolidiert sondern scheint zurzeit zu expandieren. ${ }^{112}$ Die Integration neuer Normen zur Legitimierung epistemischer Autorität schafft Konflikte, da

\footnotetext{
${ }^{106}$ Carton et al. 2020; Tsing et al. 2020

107 Yusoff 2018; Turnhout et al. 2013; Tsing et al. 2020

${ }^{108}$ Haraway 2016; Folkers 2020; Tsing et al. 2020

${ }^{109}$ Burke et al. 2016; Lövbrand et al. 2020

${ }^{110}$ bspw. Beck, Forsyth 2020; Esguerra, van der Hel 2021

${ }^{111}$ Kowarsch, Jabbour 2017; Haas 2017

${ }^{112}$ Maas et al. 2021
} 
etablierte Normen globaler Wissenschaft und Politik zum Teil in Frage gestellt werden. Folglich versuchen Expertenorganisationen diese unterschiedlichen Normen durch ständige 'Grenzarbeit' auszutarieren.

Um diese Autoritätsdynamiken zu greifen, bedarf es einer Konzeptualisierung von epistemischer Autorität, die die Entstehung und Erhaltung von Autorität selbst zum Forschungsgegenstand macht - epistemische Autorität wird zum Explanandum. Konflikte um institutionelle Designs werden als Verhandlungen um Selektionskriterien gedeutet, die darüber entscheiden, wer und in welcher Form Mitspracherecht erhält in der Interpretation politikrelevanten Wissens. ${ }^{113}$

Diese Überlegungen können eingebettet werden in eine politische Epistemologie des Anthropozäns, die sich neben Expertenorganisationen auch anderen Praktiken, Institutionen und Diskursen widmet, die Wissensbestände als Antwort auf existentielle Probleme liefern. Eine solche Epistemologie ist politisch, da sie die Mechanismen untersucht, die bestimmtes Wissen relevant für Politik macht und anderes ausgrenzt. Sie verweist auf das Anthropozän um der neuen erdsystemischen Relationalität Rechnung zu tragen. ${ }^{114}$

\section{Literatur}

Allan, Bentley B. 2017. »Producing the Climate: States, Scientists, and the Constitution of Global Governance Objects«, in International Organization 71, 1, S. 131-162.

Anderson, Kevin; Jewell, Jessica 2019. »Debating the Bedrock of Climate-Change Mitigation Scenarios«. Nature 573, S. 348-349.

Bäckstrand, Karin 2003. »Civic Science for Sustainability: Reframing the Role of Experts, PolicyMakers and Citizens in Environmental Governance«, in Global Environmental Politics 3, 4, S. 24-41.

Beck, Silke; Borie, Maud; Chilvers, Jason; Esguerra, Alejandro; Heubach, Katja; Hulme, Mike; Lidskog, Rolf; Lövbrand, Eva; Marquard, Elisabeth; Miller, Clark; Nadim, Tahani; Neßhöver, Carsten; Settele, Josef; Turnhout, Esther; Vasileiadou, Eleftheria; Görg, Christoph 2014.

»Towards a Reflexive Turn in Global Environmental Expertise - The Cases of the IPCC and IPBES«, in GALA 22, 2, S. 80-88.

Beck, Silke; Forsyth, Tim; Kohler, Pia M.; Lahsen, Myanna; Mahony, Martin 2017. »The Making of Global Enviornmental Science and Politics«, in The Handbook of Science and Technology Studies., hrsg. v. Felt, Ulrike; Fouché, Rayvon; Miller, Clark A.; Smith-Doerr, Laurel, S. 1059-1086.

Cambridge, MA: MIT Press.

Beck, Silke 2019. »Coproducing Knowledge and Politics of the Anthropocene: The Case of the Future Earth Program.", in Anthropocene Encounters: New Directions in Green Political Thinking, hrsg. v. Biermann, Frank; Lövbrand, Eva, S. 191-211. Cambridge University Press.

Beck, Silke; Forsyth, Tim 2020. »Who gets to Imagine Transformative Change? Participation and Representation in Biodiversity Assessments«, in Environmental Conservation 47, 4, S. 220-223.

Beck, Silke; Mahony, Martin 2018. »The Politics of Anticipation: the IPCC and the Negative Emissions Technologies Experience«, in Global Sustainability 1, S. 1-8.

\footnotetext{
${ }^{113}$ Montana 2019; Esguerra, van der Hel 2021
}

${ }^{114}$ Burke et al. 2016 
Beck, Silke; Oomen, Jeroen 2021. »Mapping the Corridor of Possibility for Climate Action What is at Stake in IPCC's Politics of Anticipation?«, in Environmental Science \& Policy, 123, S. 169178.

Biermann, Frank 2021. »The Future of 'Environmental'Policy in the Anthropocene: Time for a Paradigm Shift«, in Environmental Politics, 30, 1-2, S. 1-20.

Biermann, Frank; Möller, Ina 2019. »Rich man's solution? Climate engineering discourses and the marginalization of the Global South«, in International Environmental Agreements: Politics, Law and Economics 19, 2, S. 151-167.

Borie, Maud; Hulme, Mike 2015. »Framing Global Biodiversity: IPBES Between Mother Earth and Ecosystem Services«, in Environmental Science \& Policy 54, S. 487-496.

Burke, Anthony; Fishel, Stefanie; Mitchell, Audra; Dalby, Simon; Levine, Daniel J. 2016. »Planet Politics: A Manifesto from the End of IR«, in Millennium 44, 3, S. 499-523.

Carton, Wim; Asiyanbi, Adeniyi; Beck, Silke; Buck, Holly J.; Lund, Jens F. 2020. »Negative Emissions and the Long History of Carbon Removal«, in Wiley Interdisciplinary Reviews: Climate Change, S. e671.

Colgan, Jeff; Green, Jessica F.; Hale, Thomas 2021. »Asset Revaluation and the Existential Politics of Climate Change«, in International Organization, S. 586-610.

Crutzen, Paul J.; Stoermer, Eugene F. 2000. »Global Change Newsletter«, in The Anthropocene 41, S. 17-18.

Díaz, Sandra; Demissew, Sebsebe; Carabias, Julia; Joly, Carlos; Lonsdale, Mark; Ash, Neville; Larigauderie, Anne; Adhikari, Jay Ram; Arico, Salvatore; Báldi, András; Bartuska, Ann; Baste, Ivar Andreas; Bilgin, Adem; Brondizio, Eduardo; Chan, Kai MA; Figueroa, Viviana Elsa; Duraiappah, Anantha; Fischer, Markus; Hill, Rosemary; Koetz, Thomas; Leadley, Paul; Lyver, Philip; Mace, Georgina M.; Martin-Lopez, Berta; Okumura, Michiko; Pacheco, Diego; Pascual, Unai; Pérez, Edgar Selvin; Reyers, Belinda; Roth, Eva; Saito, Osamu; Scholes, Robert John; Sharma, Nalini; Tallis, Heather; Thaman, Randolph; Watson, Robert; Yahara, Tetsukazu; Hamid, Zakri Abdul; Akosim, Callistus; Al-Hafedh, Yousef; Allahverdiyev, Rashad; Amankwah, Edward; Asah, Stanley T.; Asfaw, Zemede; Bartus, Gabor; Brooks, Anathea; Caillaux, Jorge; Dalle, Gemedo; Darnaedi, Dedy; Driver, Amanda; Erpul, Gunay; Escobar-Eyzaguirre, Pablo; Failler, Pierre; Fouda, Ali Moustafa Mokhtar; Fu, Bojie; Gundimeda, Haripriya; Hashimoto, Shizuka; Homer, Floyd; Lavorel, Sandra; Lichtenstein, Gabriela; Mala, William Armand; Mandivenyi, Wadzanayi; Matczak, Piotr; Mbizvo, Carmel; Mehrdadi, Mehrasa; Metzger, Jean Paul; Mikissa, Jean Bruno; Moller, Henrik; Mooney, Harold A.; Mumby, Peter; Nagendra, Harini; Nesshover, Carsten; Oteng-Yeboah, Alfred Apau; Pataki, György; Roué, Marie; Rubis, Jennifer; Schultz, Maria; Smith, Peggy; Sumaila, Rashid; Takeuchi, Kazuhiko; Thomas, Spencer; Verma, Madhu; Yeo-Chang, Youn; Zlatanova, Diana 2015. »The IPBES Conceptual Framework-Connecting Nature and People«, in Current Opinion in Environmental Sustainability 14, S. 1-16.

Edwards, Paul N. 2010. A Vast Machine: Computer Models, Climate Data, and the Politics of Global $W$ arming. Cambridge: MIT Press.

Edwards, Paul N. 2017. »Knowledge Infrastructures for the Anthropocene«, in The Anthropocene Review 4, 1, S. 34-43.

Esguerra, Alejandro 2015. »Toward two Narratives of Knowledge«, in Innovation: The European Journal of Social Science Research 28, 1, S. 3-10.

Esguerra, Alejandro 2019. »Future Objects: Tracing the Socio-Material Politics of Anticipation«, in Sustainability Science 14, S. 963-971.

Esguerra, Alejandro; Beck, Silke; Lidskog, Rolf 2017. »Stakeholder Engagement in the Making: 
IPBES Legitimization Politics«, in Global Environmental Politics 17, 1, S. 59-76.

Esguerra, Alejandro; van der Hel, Sandra 2021. »Participatory Designs and Epistemic Authority in Knowledge Platforms for Sustainability«, in Global Environmental Politics 20, 1, S. 130-151.

Faude, Benjamin; Fuß, Julia 2020. »Coordination or Conflict? The Causes and Consequences of Institutional Cverlap in a Disaggregated World Order«, in Global Constitutionalism 9, 2, S. 268-289.

Folkers, Andreas 2020. »Was ist das Anthropozän und was wird es gewesen sein? Ein kritischer Überblick über neue Literatur zum kontemporären Erdzeitalter«, in NTM Zeitschrift für Geschichte der Wissenschaften, Technike und Medizin, 28, 4, S. 589-604.

Future Earth 2013. Future Earth Initial Design: Report of the Transition Team.

Future Earth 2018. Future Earth Names Inaugural Members to its Advisory Committee.

Future Earth 2021. Knowledge-Action Networks.

Gramelsberger, Gabriele; Feichter, Johann 2011. Climate Change and Policy: The Calculability of Climate Change and the Challenge of Uncertainty. Heidelberg \& New York: Springer Science \& Business Media.

Gustafsson, Karin M.; Lidskog, Rolf 2018. »Organizing International Experts: IPBES’s Efforts to Gain Epistemic Authority«, in Environmental Sociology 4, 4, S. 445-456.

Haas, Peter M. 1992. »Introduction: Epistemic Communities and International Policy Coordination", in International Organization 46, 1, S. 1-35.

Haas, Peter M. 2017. »The Epistemic Authority of Solution-Oriented Global Environmental Assessments«, in Environmental Science \& Policy 77, S. 221-224.

Haas, Peter M. 2018. Preserving the Epistemic Authority of Science in World Politics. WZB discussion Paper.

Hamilton, Clive 2017. Defiant Earth: The Fate of Humans in the Anthropocene. Cambridge, Malden: John Wiley \& Sons.

Haraway, Donna J. 1988. »Situated Knowledges: The Science Question in Feminism and the Privilege of Partial Perspective«, in Feminist studies 14, 3, S. 575-599.

Haraway, Donna J. 2016. Staying with the trouble: Making kin in the Chthulucene. Durham: Duke University Press.

van der Hel, Sandra 2016. »New Science for Global Sustainability? The Institutionalisation of Knowledge Co-Production in Future Earth«, in Environmental Science \& Policy 61, S. 165-175.

Hughes, Hannah; Vadrot, Alice 2019. »Weighting the World: IPBES and the Struggle over Biocultural Diversity«, in Global Environmental Politics 19, 2, S. 14-37.

Jasanoff, Sheila 2004. States of Knowledge: the Co-Production of Science and Social Order. London: Routledge.

Jasanoff, Sheila 2006. »Judgment under siege: The three-body problem of expert legitimacy«, in Democratization of Expertise? Exploring Novel Forms of Scientific Advice in Political DecisionMaking, hrsg. v. Maasen, Sabine; Weingart, Peter, S. 209-224. Dordrecht: Springer.

Keller, Ann C. 2010. »Credibility and Relevance in Environmental Policy: Measuring Strategies and Performance Among Science Assessment Organizations«, in Journal of Public Administration Research and Theory 20, 2, S. 357-386.

Kershaw, Eleanor Hadley 2018. »Leviathan and the hybrid network: Future Earth, co-production and the experimental life of a global institution «, in Science and the politics of openness, hrsg. v. 
Hartley, Sarah et al., S. 107-130. Manchester: Manchester University Press

Knappe, Henrike; Holfelder, Anne-Katrin; Löw Beer, David; Nanz, Patrizia 2019. »The Politics of Making and Unmaking (Sustainable) futures: Introduction to the Special Feature«, in Sustainability Science 14, 4, S. 891-898.

Kowarsch, Martin; Jabbour, Jason 2017. »Solution-oriented global environmental assessments: Opportunities and challenges«, in Environmental Science \& Policy 77, S. 187-192.Krisch, Nico 2017. »Liquid Authority in Global Governance«, in International Theory 9, 2, S. 237-260.

Latour, Bruno 2018. Down to Earth: Politics in the New Climatic Regime. Cambridge: John Wiley \& Sons.

Leemans, Rik 2016. »The Lessons earned from Shifting from Global-Change Research Programmes to Transdisciplinary Sustainability Science«, in Current Opinion in Environmental Sustainability 19, S. 103-110.

Lövbrand, Eva; Mobjörk, Malin; Söder, Rickard 2020. »The Anthropocene and the Geo-Political Imagination: Re-Writing Earth as Political Space«, in Earth System Governance, S. 100051.

Maas, Timo Y.; Montana, Jasper; van der Hel, Sandra; Kowarsch, Martin; Tuinstra, Willemijn; Schoolenberg, Machteld; Mahoney, Martin; Lucas, Paul L.; Kok, Marcel; Bakkes, Jan; Turnhout, Esther 2021. »Effectively Empowering: A Different Book at bolstering the Effectiveness of Global Environmental Assessments«, in Environmental Science \& Policy 123, S. 210-219.

Miller, Clark A. 2017. »Engaging with Societal Challenges«, in The Handbook of Science and Technology Studies., hrsg. v. Felt, Ulrike; Fouché, Rayvon; Miller, Clark A.; Smith-Doerr, Laurel, S. 909-913. Cambridge, MA: MIT Press.

Miller, Clark A.; Wyborn, Carina 2018. »Co-Production in Global Sustainability: Histories and Theories«, in Environmental Science \& Policy, S. in press.

Mitchell, Ronald Bruce; Clark, William C.; Cash, David W.; Dickson, Nancy M. 2006. Global Environmental Assessments. Cambridge, MA.: MIT Press.

Montana, Jasper 2019. »Co-Production in Action: Perceiving Power in the Organisational Dimensions of a Global Biodiversity Expert Process«, in Sustainability Science 14, 6, S. 1581-1591.

Moore, Jason W. 2015. Capitalism in the Web of Life: Ecology and the Accumulation of Capital. Verso Books.

Nadim, Tahani 2016. »Biodiversität erfassen: von Suppen und Satelliten«, in Diversität: Geschichte und Aktualität eines Konzepts, hrsg. v. Barras, Vincent; Blum, André; Zschocke, Nina; Rheinberger, Hans-Jörg, Würzburg: Königshausen \& Neumann.

Nature 2010. »Wanted: an IPCC for Biodiversity«, in Nature 465, 7298, S. 525-525.

Pielke, Roger 2018. »Opening up the Climate Policy Envelope«, in Issues in Science and Technology 34, 4, S. 30-36.

Plurinational State of Bolivia 2014. Living-well in balance and harmony with Mother Earth. A proposal for establishing a new global relationship between human beings and Mother Earth https://www.genevaenvironmentnetwork.org/wp-content/uploads/2020/05/livingwell_pdf.pdf (Zugriff vom 21.7.2021).

Renn, Jürgen; Scherer, Bernd 2015. Das Anthropozän: Zum Stand der Dinge. Berlin: Matthes \& Seitz. Scheffer, Thomas im Erscheinen. »Existentielle Probleme, soziologisch«, in Zeitschrift für Theoretische Soziologie.

Seawright, Jason; Gerring, John 2008. »Case Selection Techniques in Case Study Research: A 
Menu of Qualitative and Quantitative Options«, in Political research quarterly 61, 2, S. 294-308.

Sending, Ole J. 2015. The Politics of Expertise: Competing for Authority in Global Governance. Ann Arbor: University of Michigan Press.

Sending, Ole Jacob 2017. »Recognition and liquid authority«, in International Theory 9, 2, S. 311 328.

Straßheim, Holger 2015. »Politics and Policy Expertise«, in Handbook of Critical Policy Studies, hrsg. v. Fischer, Frank; Torgerson, Douglas; Durnová, Anna; Orsini, Michael, Cheltenham UK: Edward Elgar.

Tsing, Anna Lowenhaupt 2015. The Mushroom at the End of the World: On the Possibility of Life in Capitalist Ruins. Princeton: Princeton University Press.

Tsing Lowenhaupt, Anna; Deger, Jennifer; Saxena Keleman, Alder; Zhou, Feifei 2020. »What is the Anthropocene? «, in Feral Atlas: The More-than-Human Anthropocene, S. online. Stanford: Stanford University Press.

Turnhout, Esther; Bloomfield, Bob; Hulme, Mike; Vogel, Johannes; Wynne, Brian 2012. »Conservation policy: Listen to the voices of experience«, in Nature 488, 7412, S. 454-455.

Turnhout, Esther; Waterton, Claire; Neves, Katja; Buizer, Marleen 2013. »Rethinking biodiversity: from goods and services to "living with" "«, in Conservation Letters 6, 3, S. 154-161.

WBGU 2011. Welt im Wandel. Gesellschaftsvertrag für eine Große Transformation. Berlin.

Yusoff, Kathryn 2018. A Billion lack. Anthropocenes or None. Minneapolis: University of Minnesota Press.

Zürn, Michael 2018. A Theory of Global Governance: Authority, Legitimacy, and Contestation. Oxford: Oxford University Press.

\title{
Zusammenfassung
}

Angesichts existentieller Probleme - bspw. Klimawandel - entwirft der Artikel eine politische Epistemologie des Anthropozäns und zeigt diese anhand epistemischer Autorität globaler Expertenorganisationen. Um Autoritätsdynamiken zu greifen, wird ein prozessuales Verständnis epistemischer Autorität entwickelt und an drei prominenten Expertenorganisationen (Future Earth, IPBES, IPCC) illustriert.

Schlagwörter: Anthropozän, Epistemische Autorität, Wissenschaft, Global Governance Expertenorganisationen, Future Earth, IPBES, IPCC.

\begin{abstract}
In the presence of existential problems such as climate change, this article outlines a political epistemology of the Anthropocene; it focuses on how global expert organizations innovate their design resulting in conflicts over legitimizing epistemic authority. I develop a procedural understanding of authority illustrating this with three prominent expert organizations (Future Earth, IPBES, IPCC).
\end{abstract}

Key Words: Anthropocene, epistemic authority, science, global governance, expert organisationen, Future Earth, IPBES, IPCC. 
Autorenangaben

Bielefeld Universität

Fakultät für Soziologie

PF 100131 | 33501 Bielefeld

Alejandro.esguerra@uni-bielefeld.de 